\title{
LA EDUCACIÓn PARA LA CIUDADAnÍA GLOBAL EN TIEMPOS DE CRISIS
}

\author{
Education for Global Citizenship in Times \\ of Crisis
}

\begin{abstract}
Alberto Medina
Entreculturas

E-mail: a.medina@entreculturas.org

La actual crisis de la COVID-19 está suponiendo un serio obstáculo para el cumplimiento de la Agenda 2030 con la previsión de aumento de la tasa de pobreza mundial y revirtiendo muchos de los logros conseguidos en estos últimos años. Igualmente, la pandemia está conllevando el retroceso en el acceso al derecho a una educación de calidad y un aumento de la desigualdad de oportunidades generada por la brecha digital, afectando a millones de estudiantes que han dejado de ir a la escuela. Sin embargo, a pesar de todas las amenazas que despierta el actual contexto se vislumbran las oportunidades que están surgiendo en esta crisis. El cumplimiento de los ODS, sin duda, es una estrategia fundamental para afrontarla y en este sentido la educación para la ciudadanía global es una herramienta clave para acompañar procesos en los que se fomente la construcción de una ciudadanía activa, crítica y generadora de cambios sociales.

The current crisis of COVID-19 is posing a serious obstacle to the fulfilment of the 2030 Agenda, with the forecast of an increase in the world poverty rate, reversing many of the achievements made in recent years. Likewise, the pandemic is leading to a decline in access to the right to quality education and an increase in the inequalities of opportunities generated by the digital divide, affecting millions of students who have stopped going to school. However, despite all the threats aroused by the current context, the opportunities that are emerging in this crisis are glimpsed. Compliance with the SDGs is undoubtedly a fundamental strategy to face it and, in this sense, education for global citizenship is a key tool to accompany processes that promote the construction of an active, critical and generator of social change citizenship.
\end{abstract}

Educación para la ciudadanía global; derecho a la educación; Agenda 2030; Objetivos de Desarrollo Sostenible; ODS; crisis; COVID-19; agentes de transformación social.

Key words

Global citizenship education; education rights; 2030 Agenda; Sustainable Development Goals;

SDGs; crisis; COVID-19; agents of social transformation. 


\section{La Agenda 2030 ante el reto de afrontar el contexto actual de crisis}

El contexto actual de crisis provocado por la COVID-19 ha acentuado y complejizado mucho más si cabe el cumplimiento de los retos que a nivel global se venían planteando en estas dos últimas décadas y que estaban recogidos primero en los Objetivos de Desarrollo del Milenio (ODM) y a partir del 2015 en la Agenda 2030 o los denominados Objetivos de Desarrollo Sostenible (ODS).

El cumplimiento de estos nos hubiera dado muchas certezas, estrategias y herramientas para poder afrontar la crisis desde el aspecto sanitario, económico y educativo. "Si hubiéramos de verdad invertido en los ODM (Objetivos de Desarrollo del Milenio) del año 2000 y en los ODS, hubiéramos tenido una base mucho más fuerte para resistir estos choques” (López Escorial, 2020).

Por ello, el riesgo de no cumplirlos nos llevaría, y ya nos está llevando, a un panorama desolador. Según el Banco Mundial (2020):

Por primera vez desde 1998, las tasas de pobreza aumentarán a medida que la economía mundial entra en recesión. La crisis revertirá casi todos los avances logrados en los últimos cinco años y entre 40 millones y 60 millones de personas caerán en la pobreza extrema, en comparación con los datos de 2019.

A su vez desde la ONU, su vicepresidenta general señala al respecto:

Nadie será capaz de superar esta situación por sí solo. Para que todas las personas salgan reforzadas de la pandemia de la COVID-19 será necesaria una extraordinaria muestra de solidaridad o nos arriesgamos a que grandes cantidades de población queden más rezagadas. Cualquier aumento de esta brecha representa una amenaza de que la gente caiga en la pobreza, perdiendo los logros que tanto les costó conseguir y debilita nuestros sistemas para responder a la siguiente emergencia. (Mohammed, 2020)

Ante esta compleja situación, el contexto nos confronta con la necesidad urgente de tejer una mirada y una estrategia global, superando las prioridades localistas bajo la lógica del sálvese quien pueda. Sin duda es una oportunidad, a pesar de las múltiples y evidentes amenazas, de aprovechar la Agenda 2030 para que forme parte de la estrategia de reconstrucción durante el periodo post-COVID-19, afrontando una crisis sistémica como la que estamos atravesando a través de una agenda sistémica como la que plantean, o vocacionalmente pretenden plantear, los Objetivos de Desarrollo Sostenible.

\section{Panorama actual del derecho a la educación}

Este contexto de crisis está teniendo un impacto negativo en el derecho a la educación y millones de niños y niñas en todo el mundo están siendo afectados y afectadas. Según datos de la UNESCO, "290 millones de estudiantes han perdido ya sus clases debido a las medidas para contener la propagación de la epidemia” (UNICEF, 2020).

La dificultad en el acceso al derecho a una educación de calidad genera desigualdad de oportunidades que aumenta más si cabe por la brecha digital. Este es uno de los elementos de mayor relevancia a la hora de abordar el derecho a la educación dentro del contexto de la pandemia, aunque no el único. "No todos los alumnos pueden acceder a los contenidos y servicios edu-
El contexto actual de crisis provocado por la COVID-19 ha acentuado y complejizado mucho más si cabe el cumplimiento de los retos que a nivel global se venían planteando en estas dos últimas décadas 
cativos digitales en condiciones de igualdad y calidad, agravándose la brecha digital educativa, particularmente entre alumnado con bajos recursos, inmigrantes y discapacitados; perjudicando el principio de la igualdad de oportunidades" (Navarro, 2020).

La brecha de aprendizaje se ensancha cuando hablamos de menores en situación de desplazamiento o refugio. Según ACNUR hay más de un 35\% de niños y niñas que no tienen acceso a la educación primaria debido en gran parte a la compleja situación que se vive en los campos de personas refugiadas.

Esta crisis ha constatado que la educación a distancia a través de la tecnología digital no es la solución, al menos no la única, sino que por el contrario puede agrandar la brecha entre los y las estudiantes que viven en contextos de especial vulnerabilidad. "Más de la mitad de la población estudiantil del mundo no tiene una computadora en casa y el 43\% (más de 700 millones) no tiene acceso a internet" (International Telecommunication Union (ITU), 2020).

A su vez esta situación también queda reflejada en España según los datos del último informe PISA de la OCDE, donde, mientras el $61 \%$ del alumnado de las familias más aventajadas tienen tres o más ordenadores en casa, el $44 \%$ de las desaventajadas tienen solo uno y el $14 \%$ ninguno.

Según UNESCO, la reducción del tiempo de enseñanza influye en el rendimiento escolar, y las familias con más recursos pueden utilizarlos para compensar la pérdida de clases. Save the Children analiza en el informe COVID, cerrar la brecha el impacto de la medida del cierre en los centros escolares: "Este cierre ha conllevado limitaciones en las oportunidades de aprendizaje del alumnado, especialmente el más vulnerable" (Save the children, 2020).

Además de la falta y la dificultad en el acceso a la educación, el informe La Solidaridad para relanzar la educación, destaca lo siguiente:

No solo es un tema de acceso a conocimientos sino también de niveles de atención, seguimiento y acompañamiento. La educación promueve el desarrollo social, emocional y cognitivo de niños y niñas. Los niños que se sienten valorados y aceptados mantienen relaciones saludables y generan mayor autonomía y seguridad. (Entreculturas, 2020)

Por ello, para fortalecer el desarrollo social, emocional y cognitivo en la infancia es importante generar entornos de cuidado y acompañamiento que potencien estas capacidades. Precisamente este ha sido otro de los déficits que ha planteado la COVID-19: la dificultad en la atención y el cuidado de la infancia. A este respecto el trabajo de cuidados ha recaído y recae mayoritariamente entre mujeres y niñas que ven mermadas las oportunidades para su desarrollo tanto educativo como profesional.

También se constata, en este sentido, cómo las jóvenes y las niñas también aparecen dentro de los sectores más vulnerables de la población ante las crisis. En otras epidemias se ha constatado cómo aumenta tanto el número de embarazos no deseados, la violencia ejercida contra ellas, así como el porcentaje de absentismo de jóvenes y niñas, entre otros aspectos.

Aplicando una perspectiva más inclusiva sería necesario vincular el ODS 4 (educación) y el ODS 5 (género) a la hora de trazar una estrategia que busque reducir esta brecha de desigualdad. Como señala ONU mujeres: "Responder a la pandemia no solo requiere rectificar desigualdades históricas, sino también construir un mundo resiliente para el interés de todas las personas, con las mujeres como sujeto de recuperación” (ONU Mujeres, 2020).

\section{La dificultad en el acceso al derecho a una educación de calidad genera desigualdad de oportunidades que aumenta más si cabe por la brecha digital}




\section{La educación para la ciudadanía global como enfoque transformador}

El derecho a la educación como un derecho fundamental otorga y legitima a los Estados a ser garantes de los servicios básicos que lo articulan, implicando por tanto una función esencial para la construcción de ciudadanía. Por ello este punto de vista de enfocar el derecho, tanto desde lo local como en lo global, supone un "principio básico de ciudadanía”.

Así lo determina la meta 4.7, dentro del ODS 4 UNESCO (2015),

Garantizar que todos los alumnos adquieran los conocimientos teóricos y prácticos necesarios para promover el desarrollo sostenible, entre otras cosas mediante la educación para el desarrollo sostenible y la adopción de estilos de vida sostenibles, los derechos humanos, la igualdad entre los géneros, la promoción de una cultura de paz y no violencia, la ciudadanía mundial y la valoración de la diversidad cultural y de la contribución de la cultura al desarrollo sostenible, entre otros medios.

De esta forma, la educación para la ciudadanía global nos permite tomar distancia sobre nuestra realidad local y sin olvidarnos de ella, ganar altura para tener una mirada más amplia y compleja de la realidad que nos rodea. Se nos plantea guardar un equilibrio entre nuestra realidad local, nuestro contexto más cercano y sobre el que podremos ejercer mayor influencia; y la realidad global, aquella que nos resulta más lejana y apartada de nuestra cosmovisión del mundo. Replantearnos la mirada y guardar este equilibrio nos hará ser agentes de transformación en los diferentes planos de la realidad. En un contexto de crisis como el que sufrimos es vital y necesaria esta mirada que nos haga ser personas responsables con las realidades y contextos complejos que nos ha tocado vivir.

Cuando hablamos de ciudadanía global, también supone hablar de una mirada global en cuanto al enfoque en derechos y su necesaria interdependencia tanto en el cumplimiento como en el incumplimiento de estos.

La Fundación Entreculturas, junto con otras entidades del sector, entiende la educación para la ciudadanía global como una herramienta que facilita esa mirada, para posteriormente construir propuestas transformadoras y de cambio social. Por ello urge trazar alianzas y trabajar en red desde las diferentes organizaciones del sector y con los agentes sociales a través de los distintos territorios, para intensificar ahora más que nunca, procesos de educación para la ciudadanía.

En este sentido la Coordinadora Estatal de ONG en su documento 20 medidas para hacer frente a la pandemia del COVID-19: desde el sistema de cooperación para el desarrollo y las ONGD defiende lo siguiente (Coordinadora Estatal ONG, 2020),

En materia de Educación para la Ciudadanía Global (EpCG), es necesario un compromiso de la Agencia Estatal de Cooperación al Desarrollo para apoyar la continuidad y refuerzo del trabajo de Educación para el Desarrollo y la Ciudadanía Global durante y de manera posterior a la emergencia para multiplicar y extender la promoción de los valores de la solidaridad, cooperación y el internacionalismo entre la sociedad española. Estas son herramientas claves en la lucha contra la pandemia y por la justicia global. En este sentido, es importante promover una mayor inversión en educación para el desarrollo sostenible y la ciudadanía global, de manera articulada con la cooperación descentralizada y con otros ministerios competentes (Educación, MITECO, Derechos Sociales, Igualdad), en el marco de la Agenda 2030 y alineada con la meta 4.7.
La educación para la ciudadanía global nos permite tomar distancia sobre nuestra realidad local y sin olvidamos de ella, ganar altura para tener una mirada más amplia y compleja de la realidad que nos rodea 
A modo de recogida sobre lo ya señalado, la actual de crisis de la COVID-19 ha puesto en evidencia la dificultad de seguir sosteniendo una educación ajena a la realidad global en la que vivimos, tanto en los contenidos tratados como en las habilidades fomentadas. El escenario global en el que vivimos y que va a quedar a consecuencia de esta crisis es complejo, y una respuesta responsable al mismo va a requerir el fomento de conocimientos y habilidades para la promoción de una ciudadanía corresponsable, que sea capaz de vivir su vida de forma interconectada con otros y otras, en la tarea de contribuir en la configuración de escenarios locales y globales más justos y solidarios. Si no asumimos la centralidad de la educación en estos momentos y no apostamos por un enfoque educativo que sea capaz de atender a esta necesidad, estaremos dejando sin herramientas a las futuras generaciones, formadas solo en el ámbito académico y para el ejercicio de profesiones, en un futuro global cada vez más incierto y complejo que necesita una ciudadanía implicada con la construcción de un mundo más justo.

\section{Propuestas de transformación social}

Como vimos, la educación para la ciudadanía global tiene un gran potencial transformador, entendida como una herramienta a corto, medio y largo plazo para abordar tanto la actual crisis global, como los retos globales que nos interpelarán en el futuro.

Centrándonos en el corto y medio plazo, queríamos abordar la cuestión de qué puede aportar la Ciudadanía Global en este contexto de crisis de COVID-19. Compartimos en este sentido algunas reflexiones:

» Desde la herencia que la ciudadanía global ha recogido, sin duda, de la educación popular y los postulados de Paolo Freire, el formar agentes de transformación social pasa por fomentar una educación que facilite experiencias para fortalecer una ciudadanía informada, crítica, comprometida, activa y conectada con los retos globales de nuestro tiempo, y en particular con los retos que plantea la crisis de la COVID-19.

» Para construir una ciudadanía consciente de los desafíos globales, con capacidad de abordar la prevalente desigualdad y exclusión social, y responder a la crisis de la COVID-19, es urgente fortalecer a la comunidad educativa y su articulación con los barrios, fomentando la participación de la juventud en el cumplimiento de la Agenda 2030. Siguiendo recomendaciones del VII Informe Foessa 2019, apostamos por proyectos participativos que promuevan la convivencia intercultural, inclusión social y la creación de redes educativas de solidaridad para abordar estos desafíos.

» Una ciudadanía capaz de articularse con otras personas y colectivos en todo el mundo a través de la pedagogía del encuentro y la creación de redes, que incida en lo local para transformar lo global. Promoviendo en ambos ámbitos las relaciones justas y equitativas entre las personas y con el planeta, para así garantizar la supervivencia de ambos.

» Una educación que acompañe a las personas durante el proceso educativo y facilite herramientas, habilidades y recursos concretos para actuar sobre la realidad. Destacando el trabajo en emociones para poder reconocerlas y actuar, teniendo capacidad para tomar decisiones generadoras de cambios sociales.

» Si hablamos de ciudadanía global, debemos hablar de la necesidad de fomentar una cultura de la convivencia y de paz y de la cohesión de sociedades interculturales basadas en los derechos humanos y en una cultura de la hospitalidad que genere espacios de diálogo y aco-

\section{La educación para la ciudadanía global tiene un gran potencial transformador, entendida como una herramienta a corto, medio y largo plazo para abordar tanto la actual crisis global, como los retos globales que nos interpelarán en el futuro}


gida, haciendo hincapié en los contextos de mayor exclusión y en las personas que presentan mayor grado de vulnerabilidad.

En relación con estas últimas intuiciones queríamos compartir algunas propuestas educativas concretas que desde Entreculturas estamos realizando desde el enfoque de la educación para la ciudadanía global:

La Red Solidaria de Jóvenes. (RSJ) Es un programa de participación social de jóvenes en centros e instituciones socioeducativas, dirigido a adolescentes de entre 12 y 18 años — que participan de manera voluntaria-, basado en el trabajo social con grupos de iguales. El programa sigue un itinerario en el que se ofrecen espacios de reflexión y análisis de diferentes realidades de desigualdad y exclusión, para posteriormente generar acciones desde los contextos locales, pero con una perspectiva global. Es un programa que ofrece herramientas y recursos para potenciar las habilidades sociales como agentes de cambio.

Red Generación 21. Red internacional de jóvenes de 13 a 29 años de más de 21 países que se articulan para formar parte de una ciudadanía juvenil para la paz que logre impulsar y hacer visible su acción comprometida en la transformación de las situaciones que generen violencia y la construcción de otro mundo posible y necesario. La Red solidaria de Jóvenes pertenece a esta red internacional y es una gran oportunidad para articularse con otras personas y generar redes que supongan encuentros y aprendizajes desde la diversidad.

Programas de aprendizaje-servicio. Con esta metodología se adquieren conocimientos, habilidades y actitudes; primero a través de la puesta en marcha de aprendizajes y contenidos, curriculares o no, y en un segundo momento, una vez adquiridos los aprendizajes, el alumnado pone en marcha un proyecto basado en estos contenidos y que se desarrolle como parte del servicio a la comunidad. Hemos acompañado diferentes programas de aprendizaje-servicio con jóvenes de universidad (proyecto Miradas que migran) y con jóvenes de educación secundaria, trabajando también sobre la realidad de las migraciones forzosas.

Decide Convive. Proceso de trabajo con profesionales de la intervención educativa, que engloba docentes, dentro del ámbito educativo formal, y educadoras/es y otros agentes educativos, en el ámbito de la educación no formal. El foco de trabajo dentro de este proceso es el análisis y la reflexión en torno a la convivencia, partiendo de un itinerario más personal, lo que supone la convivencia para cada participante, hasta cómo se vive en cada uno de los entornos profesionales. El proceso propone una serie de herramientas que puedan facilitar, acompañar y potenciar los diferentes procesos de convivencia que se lleven a cabo. También supone el intercambio de experiencias y aprendizajes entre diferentes profesionales de la intervención socioeducativa.

Procesos de pedagogía teatral. El teatro puesto al servicio del ámbito educativo se revela como una potente herramienta para trabajar diferentes habilidades sociales, comunicativas y de expresión, potenciando la educación y la gestión emocional. Esta herramienta en conexión con la educación para la ciudadanía global permite a las personas que participan en estos procesos, fundamentalmente jóvenes, profundizar en el trabajo de empoderamiento como ciudadanía responsable y agentes de cambio. Una metodología muy emparentada con la educación popular y el trabajo desarrollado por Augusto Boal y el Teatro del oprimido y que años más tarde nos ha permitido publicar Un mundo de Teatro que se centra en el trabajo de la convivencia y la cultura de paz a través de la pedagogía teatral (Entreculturas, 2018).

Para finalizar y volviendo al contexto de crisis de la COVID-19 cabe señalar que desde la Fundación Entreculturas hemos vivido estos meses con mucha incertidumbre, pero también desde
Desde la Fundación

\section{Entreculturas}

hemos vivido estos

meses con mucha incertidumbre, pero también desde

la oportunidad

de seguir

acompañando a

jóvenes, docentes

$y$ otros agentes

educativos desde

los diferentes

procesos

anteriormente

señalados 
la oportunidad de seguir acompañando a jóvenes, docentes y otros agentes educativos desde los diferentes procesos anteriormente señalados. Transitando por momentos complejos y difíciles, al ver cómo las desigualdades y las brechas sociales aumentan, y a la vez caminando por sendas llenas de aprendizajes y con la certeza de que el trabajo desde la educación para la ciudadanía global es una herramienta indispensable para poder seguir construyendo sociedades más justas e igualitarias; y a su vez siendo un canal fundamental de transmisión que fomenta e impulsa el cumplimiento de la Agenda 2030.

\section{Referencias}

Banco Mundial. (2020). Pobreza. Panorama general. Recuperado el 17/6/2020 de https://www. bancomundial.org/es/topic/poverty/overview

Coordinadora estatal ONG. (2020) La solidaridad es parte de nuestra crisis. Recuperado el 22/5/2020 de https://coordinadoraongd.org/2020/04/20-medidas-para-hacer-frente-a-lapandemia-del-covid-19/

Entreculturas. (2018). Un mundo de Teatro. Recuperado el 19/5/2020 de https:/www.entreculturas.org/es/publicaciones/un-mundo-de-teatro

Entreculturas. (2020). La Solidaridad para relanzar la educación. Recuperado el 10/6/2020 de https://escuela.entreculturas.org/2463-2/

International Telecommunication Union (ITU). (2020). https://www.itu.int/es/Pages/default. aspx

López Escorial, M. (2020). ¿Dónde quedaron los ODS y la agenda 2030? Recuperado el 9/7/2020 de https://elpais.com/elpais/2020/05/19/planeta_futuro/1589903800_174767.html

Navarro Sanz, B. (2020). El impacto de la crisis de la COVID-19 en los Objetivos de Desarrollo Sostenible: ¿un retroceso sin precedentes en la Agenda 2030? Recuperado el 17/7/2020 de ieee.es/documento de Opinión 78/2020 http://www.ieee.es/alerias/fichero/docs_opinion/2020/DIEEEO78_2020BEANAV_CovidODS.pdf

Mohammed, A. (2020). La crisis provocada del coronavirus, una oportunidad para cumplir los objetivos de desarrollo sostenible. Recuperado el 18/7/2020 de https://news.un.org/es/ story/2020/05/1474602

OCDE. (2019). Informe PISA 2019. Recuperado el 18/7/2020 de http://www.educacionyfp. gob.es/inee/publicaciones/informes.html

ONU Mujeres. (2020). Los efectos del COVID sobre las mujeres y niñas. Recuperado el 16/7/2020 de https://interactive.unwomen.org/multimedia/explainer/covid19/es/index.html

Save The Children. (2020). Informe Covid-19: cerrar la brecha. Recuperado el 20/7/2020 de https://www.savethechildren.es/sites/default/files/2020-05/COVID19Cerrarlabrecha. pdf

UNICEF. (2020). El nuevo coronavirus y el derecho a la educación. Recuperado el 15/7/2020 de https://www.unicef.es/educa/blog/nuevo-coronavirus-derecho-educacion 